\title{
miRNA-130b is required for the ERK/FOXM1 pathway activation-mediated protective effects of isosorbide dinitrate against mesenchymal stem cell senescence induced by high glucose
}

\author{
JIANFENG XU ${ }^{1-3}$, ZHEYONG HUANG ${ }^{2}$, LI LIN $^{4}$, MINGQIANG FU ${ }^{2}$, YANAN SONG ${ }^{2}$, YUNLI SHEN $^{4}$, \\ DAOYUAN REN ${ }^{2}$, YANHUA GAO ${ }^{2}$, YANGANG SU ${ }^{2}$, YUNZENG ZOU ${ }^{2,3}$, YUEGUANG CHEN ${ }^{1}$, \\ DADONG ZHANG ${ }^{1}$, WEI HU ${ }^{1}$, JUYING QIAN ${ }^{2}$ and JUNBO GE ${ }^{2,3}$
}

\begin{abstract}
${ }^{1}$ Department of Cardiology, Minhang Hospital, Ruijin Hospital Group, Shanghai Jiaotong University School of Medicine, Shanghai 201199; ${ }^{2}$ Department of Cardiology, Zhongshan Hospital, ${ }^{3}$ Institutes of Biomedical Scienses, Fudan University, Shanghai 200032; ${ }^{4}$ Department of Cardiology, Eastern Hospital, Tongji University, Shanghai 200120, P.R. China
\end{abstract}

Received July 15, 2014; Accepted September 30, 2014

DOI: 10.3892/ijmm.2014.1985

\begin{abstract}
The present study was carried out to investigate the hypothesis that organic nitrates can attenuate the senescence of mesenchymal stem cells (MSCs), a superior cell source involved in the regeneration and repair of damaged tissue. MSCs were treated with high glucose (HG) in order to induce senescence, which was markedly attenuated by pre-treatment with isosorbide dinitrate (ISDN), a commonly used nitrate, as indicated by senescence-associated galactosidase (SA- $\beta$-gal) activity, p21 expression, as well as by the mRNA levels of DNA methyltransferase 1 (DNMT1) and differentiated embryo chondrocyte expressed gene 1 (DEC1), which are senescencerelated biomarkers. It was also found that the senescent MSCs (induced by HG glucose) exhibited a marked downregulation in ERK activity and forkhead box M1 (FOXM1) expression, which was reversed by ISDN preconditioning. Of note, the inhibition of ERK phosphorylation or the downregulation of FOXM1 statistically abolished the favourable effects of ISDN. In addition, the investigation of the senescence-associated miR-130 family suggested that miR-130b mediates the beneficial effects of ISDN; it was found that the protective effects of ISDN against the senescence of MSCs were prominently reversed by the knockdown of miR-130b. Furthermore, the downregulation of ERK phosphorylation or FOXM1 expression decreased the miR-130b expression level; however, the suppression of miR-130b demonstrated no significant impact on ERK phosphorylation or FOXM1 expression. Taken together, to the best of our knowledge, the present study is the first to demon-
\end{abstract}

Correspondence to: Professor Junbo Ge or Professor Juying Qian, Department of Cardiology, Zhongshan Hospital, Fudan University, 180 Fenglin Road, Shanghai 200032, P.R. China

E-mail: gejunbo_zs@126.com

E-mail: qianjuying_zs@126.com

Key words: mesenchymal stem cell, senescence, hyperglycemia, nitrate, mechanism strate the favourable effects of ISDN against HG-induced MSC senescence, which are mediated through the activation of the ERK/FOXM1 pathway and the upregulation of miR-130b.

\section{Introduction}

Organic nitrates are a class of drugs that have been clinically used in the treatment of myocardial ischemia, as well as congestive heart failure for more than a hundred years. Nitrates release nitric oxide (NO), an endothelium-derived relaxing factor, executing a broad range of functions, including antioxidant, anti-inflammatory, antithrombotic and anti-atherogenic effects (1). Furthermore, NO has been shown to ameliorate the aging of cells (2), and small interfering RNA (siRNA) targeting endothelial NO synthase (eNOS) have been shown to reduce the anti-senescence effects of insulin (3). However, whether organic nitrates, a source of NO production, play a protective role against the senescence of bone marrow-derived mesenchymal stem cells (MSCs), which have potential for the repair of damaged tissue (4), remains to be clarified.

We have previously reported that MSCs are a superior stem cell source for cellular treatment and biomedical engineering due to their unique paracrine and immunosuppressive properties (5). However, cell aging, which can be induced by various pathophysiological states, including high glucose (HG), oxidative stress and inflammatory attack, can severely impair the therapeutic potential of MSCs in tissue regerenation (6). Therefore, the investigation of possible interventions to attenuate MSC senescence and the further investigation of the potential mechanisms involved is of critical clinical significance for patients with diabetes accompanied by organ injury, currently awaiting the regeneration of 'viable' MSCs $(7,8)$.

The results of previous studies have led us to hypothesize that organic nitrates may prevent the onset of MSC senescence. Lauer et al (9) demonstrated that the lack of nitrite in the plasma was mainly responsible for the inability of exercise to reverse age-dependent endothelial dysfunction. Ward et al (10) also noted that the overexpression of eNOS, which results in endogenous NO production, boosted the capability of bone marrow 
cells, leading to a substantial improvement in left ventricular ejection fraction at 6 weeks. Accordingly, in the present study, we examined the effects of isosorbide dinitrate (ISDN), a commonly used organic nitrate, on the senescence of MSCs triggered by HG. The characteristics of cellular aging, such as increased expression of senescence-associated galactosidase (SA- $\beta$-gal), cell enlargement and the upregulation of p21 expression, were investigated. In addition, the mRNA expression of the typical senescence-associated cellular biomarkers (11), including DNA methyltransferase 1 (DNMT1) and differentiated embryo chondrocyte expressed gene 1 (DEC1) was detected to characterize growth arrest. We also investigated the underlying mechanisms responsible for the effects of ISDN based on the knowledge of the key roles of ERK phosphorylation (12) and forkhead box M1 (FOXM1) regulation (13) in cell cycle modulation, and further clarified the association between them. Considering that cellular senescence has recently been implicated in regulation of microRNAs (miRNAs or miRs) (14), crucial factors of diverse pathophysiological processes, we wished to explore the potential role of senescence-associated miRNAs in the effects of ISDN on MSC senescence.

\section{Materials and methods}

All animal treatments were performed in strict accordance with the Guidelines for the Care and Use of Laboratory Animals published by the National Academy Press (NIH Publication no. 85-23, revised 1996). The study was approved by the Animal Care and Use Committee of Zhongshan Hospital, Fudan University.

Isolation and culture of MSCs. Bone marrow-derived MSCs were purified from 4-week-old male Sprague-Dawley (SD) rats as previously described (15). Briefly, the bone marrow in the tibias and femurs was flushed using Dulbecco's modified Eagle's medium (DMEM; Gibco, Grand Island, NY, USA). Following incubation in DMEM supplemented with $10 \%$ fetal bovine serum (FBS; Gibco) at $37^{\circ} \mathrm{C}$ in an atmosphere of $5 \% \mathrm{CO}_{2}$ for $24 \mathrm{~h}$, the bone marrow solution was discarded, and the adherent cells were subsequently cultured. Subsequently, the spindle-shaped, adherent MSCs were isolated and expanded, and the culture medium was regularly changed every 3-4 days. Passage 4 MSCs, which were previously demonstrated to express typical MSC-related cell surface antigens (15), were used in the subsequent experiments.

Experimental manipulation of MSCs. Native MSCs were starved for serum and glucose overnight and then exposed to medium with HG or ISDN (both from Sigma-Aldrich, St. Louis, MO, USA) at the indicated concentrations or mannitol (33 mM) for $24 \mathrm{~h}$. The untreated MSCs served as the control group and those exposed to mannitol served as the negative control group. To examine the effects of ISDN on cell aging, the starved MSCs were treated with ISDN $(50 \mu \mathrm{M})$ for $6 \mathrm{~h}$ prior to $\mathrm{HG}$ treatment. To explore the underlying mechanisms responsible for the effects of ISDN, the MSCs were incubated with PD98059 (ERK inhibitor, $20 \mu \mathrm{M}$; Santa Cruz Biotechnology Inc., Santa Cruz, CA, USA) or DMSO (dimethyl sulfoxide; Sigma-Aldrich) for $30 \mathrm{~min}$ prior to ISDN treatment or were subjected to FOXM1 knockdown using siRNA, followed by subsequent treatment with ISDN and HG.
Western blot analysis. After the indicated treatments, the MSCs were lysed in RIPA lysis buffer containing $50 \mathrm{mM}$ Tris- $\mathrm{HCl}$ (pH 7.4), 1\% NP-40, $1 \mathrm{mM}$ phenylmethylsulphonyl fluoride, $150 \mathrm{mM} \mathrm{NaCl}, 1 \mathrm{mM}$ EDTA and 1\% sodium deoxycholate. Protein lysates were separated by $10 \%$ SDS-PAGE and transferred onto polyvinylidene fluoride membranes. The membranes were washed 3 times with TBS (pH 7.6) buffer, soaked in 5\% non-fat dry milk for $2 \mathrm{~h}$ and incubated overnight at $4^{\circ} \mathrm{C}$ with anti-p21 (\#2947), anti-phosphorylated (p-)ERK (\#4370) (both from Cell Signaling Technology, Danvers, MA, USA; both diluted 1:1,000) and anti-FOXM1 (sc-271746; Santa Cruz Biotechnology Inc.) polyclonal antibodies (diluted 1:300), followed by incubation with a horseradish peroxidase-conjugated secondary antibody (diluted 1:5,000) for $2 \mathrm{~h}$ at room temperature. The immune complexes were visualized using enhanced chemiluminescence detection reagents, and the band intensity was measured, quantified and analyzed using an Image System (Bio-Rad, Hercules, CA, USA). The $\beta$-actin (\#8457; Cell Signaling Technology) band intensity served as the control for p21 and FOXM1 expression; similarly, total-ERK (\#4695; Cell Signaling Technology) served as the control for $\mathrm{p}$-ERK expression.

Reverse transcription-quantitative (real-time) polymerase chain reaction $(R T-q P C R)$. Total RNA was isolated from the cultured MSCs using TRIzol reagent (Invitrogen, Carlsbad, CA, USA). Spectrophotometric $\mathrm{OD}_{260}$ measurements were used to determine the $2 \mu \mathrm{g}$ RNA input for cDNA synthesis and cDNA was generated using a High Capacity cDNA Reverse Transcription kit (Applied Biosystems, Foster City, CA, USA) as recommended by the manufacturer. Real-time PCR was performed using the SYBR ${ }^{\circledR}$ ExScript $^{\mathrm{TM}}$ PCR kit (Takara Biochemicals, Kyoto, Japan) in a total volume of $10 \mu \mathrm{l}$ in a Bio-Rad iQ5 optical module. The primer sequences used for the genes (DNMT1,DECl and $\beta$-actin) were synthesized and are listed as follows: DNMT1 forward, 5'-GTG AAG GAG AAA TTG AAT CTC TT-3' and reverse, 5'-GAG GAA GCT GCT AAG GAC-3'; DEC1 forward, 5'-CCA GGA AAC CAT TGG ACT CAG -3' and reverse, 5'-AGA GGT CGG ATA CCA GCA TTT-3'; $\beta$-actin forward, 5'-CCA TTG AAC ACG GCA TTG-3' and reverse, 5'-TAC GAC CAG AGG CAT ACA-3'. The PCR amplification consisted of 40 cycles $\left(95^{\circ} \mathrm{C}\right.$ for $5 \mathrm{sec}, 59.5^{\circ} \mathrm{C}$ for $30 \mathrm{sec}$ ) following an initial denaturation at $95^{\circ} \mathrm{C}$ for $30 \mathrm{sec}$. Melting curves were obtained at the end of the reaction by stepwise increases in temperature of $1^{\circ} \mathrm{C} / \mathrm{min}$ from 59.5 to $95^{\circ} \mathrm{C}$ over a period of $35 \mathrm{~min}$. The threshold cycle $(\mathrm{Ct})$ value was defined as the fractional cycle number at which the fluorescence passed a fixed threshold. The fold -change in target mRNA expression was calculated using the $2^{-\Delta \Delta \mathrm{Ct}}$ method following normalization to $\beta$-actin expression.

For microRNA detection, total RNA was extracted from the MSCs using the mirVana ${ }^{\mathrm{TM}}$ miRNA Isolation kit (Ambion, Austin, TX, USA) according to the manufacturer's instructions and reverse transcribed into cDNA using the PrimeScript RT reagent kit (Takara Biochemicals). miR-130a/b was quantified using TaqMan ${ }^{\circledR}$ MicroRNA Assay kits (Applied Biosystems) with U6 small nuclear RNA as an endogenous control. All PCR reactions were performed in triplicate.

RNA interference. FOXM1 and control siRNA (scramble) were purchased from Santa Cruz Biotechnology, Inc. After the MSCs were transfected with FOXM1 or control siRNA using 
A

p21

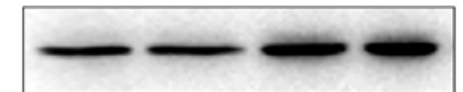

$\beta$-actin
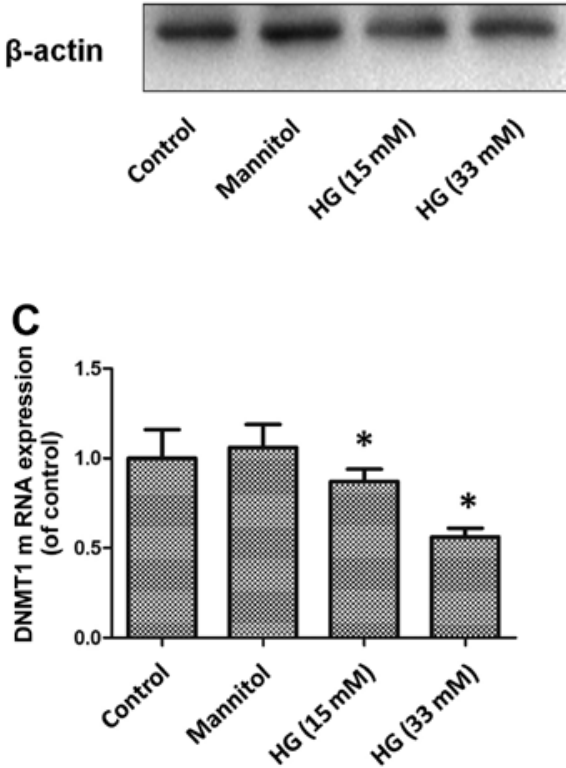

B

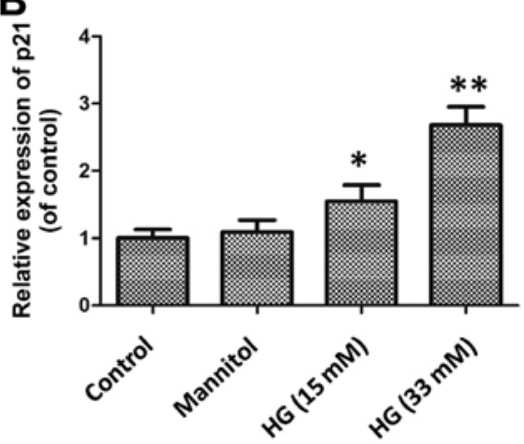

D

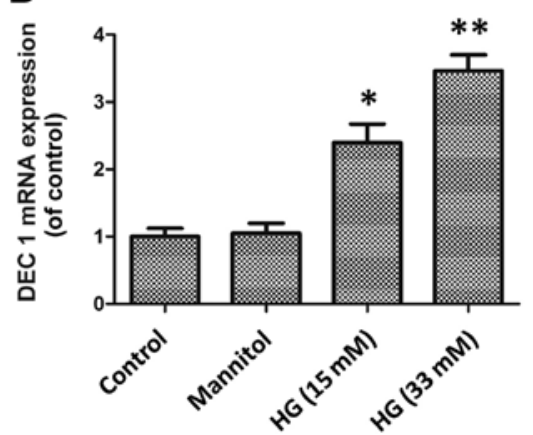

Figure 1. High glucose (HG) accelerates mesenchymal stem cell (MSC) senescence in a concentration-dependent manner. MSCs were exposed to various concentrations of $\mathrm{HG}$ for $24 \mathrm{~h}$ or to mannitol (33 mM), which served as a negative control with an osmotic pressure equal to that of $33 \mathrm{mM} \mathrm{HG}$. (A and B) Detection of p21 expression in MSCs by western blot analysis. The representative bands and statistical bar diagram are shown. The data are presented as the means \pm standard error of the mean (SEM); $\mathrm{n}=5$ in each experiment. (C and D) DNA methyltransferase 1 (DNMT1) mRNA expression and differentiated embryo chondrocyte expressed gene 1 (DEC1) mRNA expression were examined by quantitative PCR; the results are illustrated in the bar diagram. The data are presented as the means \pm SE from 5 independent experiments. ${ }^{*} \mathrm{P}<0.05,{ }^{* *} \mathrm{P}<0.01$ vs. the control group.

Lipofectamine $^{\mathrm{TM}} 2000$ (Invitrogen) as previously described by Wang et al (16), the cells were collected and processed for subsequent analysis by western blot analysis and quantitative PCR. The untreated MSCs served as the control group.

SA- $\beta$-gal assay. MSC senescence was determined based on SA- $\beta$-gal activity, which was measured using a $\beta$-galactosidase staining kit (BioVision, Palo Alto, CA, USA) following the manufacturer's instructions. Briefly, the treated MSCs were washed in PBS, fixed in $0.5 \mu \mathrm{l}$ of fixative solution for 10-15 min at room temperature, and incubated with the staining solution mix overnight at $37^{\circ} \mathrm{C}$. Green-stained cells and total cells were then counted under a microscope, and the percentage of $\beta$-galactosidase-positive and enlarged cells was calculated.

miR-130b silencing byantagomiR-based treatment. Chemically modified antisense oligonucleotides (antagomiR-130b; RiboBio, Guangzhou, China) have been previously used to inhibit miR-130b expression in MSCs in vitro (17). Antagomir oligonucleotides were transfected into the MSCs at doses of $100 \mathrm{nmol} / 1$ using Lipofectamine 2000 (Invitrogen) according to the manufacturer's instructions. As the controls, an unrelated negative control (NC; RiboBio) was transfected into the MSCs. Transfection efficiencies were determined by quantitative PCR, and the cells were processed for subsequent intervention or analysis on day 1 following transfection.

Statistical analysis. The results are expressed as the means \pm standard error of the mean (SEM). Statistical analysis, which included an unpaired Student's t-test for comparisons between 2 groups and analysis of variance (ANOVA) with Bonferroni's correction for multiple comparisons, was performed using SPSS software (version 14; SPSS Inc., Chicago, IL, USA). A P-value of $<0.05$ was considered to indicate a statistically significant difference.

\section{Results}

$H G$ induces senescence in MSCs. To determine the effects of HG on MSC senescence, we performed a dose-effect experiment. Upregulated p21 expression, decreased DNMT1 mRNA expression and increased DEC1 mRNA expression were used as markers to evaluate cellular senescence. Incubation with $\mathrm{HG}$ for $24 \mathrm{~h}$ significantly enhanced p21 expression in the MSCs in a concentration-dependent manner by $155.3 \%(\mathrm{P}<0.05)$ and $268.1 \%(\mathrm{P}<0.01)$ in the 15 and $33 \mathrm{mM} \mathrm{HG}$ groups, respectively, compared with the control group; however, culture with mannitol at an osmotic pressure equal to that provided by $33 \mathrm{mM}$ HG had no significant pro-senescence effects $(\mathrm{P}>0.05)$ on MSCs (Fig. 1A and B).

Quantitative PCR confirmed the effects of $33 \mathrm{mM} \mathrm{HG}$ on MSC senescence, as indicated by a $43.9 \%$ decrease $(\mathrm{P}<0.05)$ in DNMT1 mRNA expression and a $346.2 \%$ increase in DEC1 mRNA expression $(\mathrm{P}<0.01)$ compared with the control group (Fig. 1C and D). Analogously, the control, mannitol, did not have a notable effect on the mRNA expression of either DNMT1 or DEC1. Based on these results and those of a previous study (18), we selected the HG dose of $33 \mathrm{mM}$ to establish an experimental model of MSC senescence in the subsequent experiments. 
A

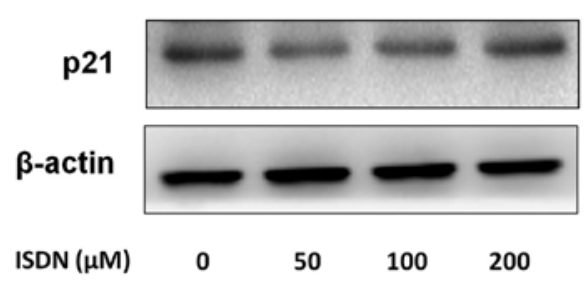

C

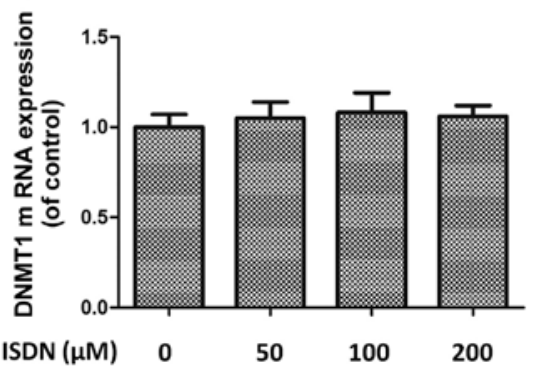

B

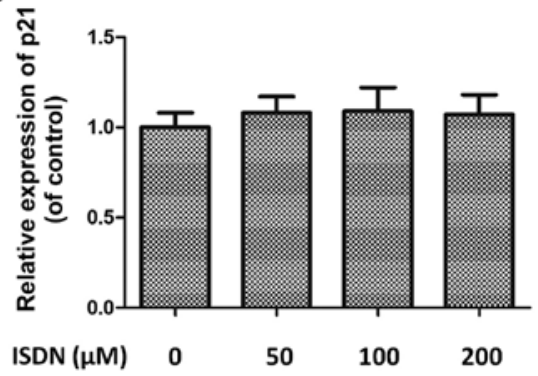

D

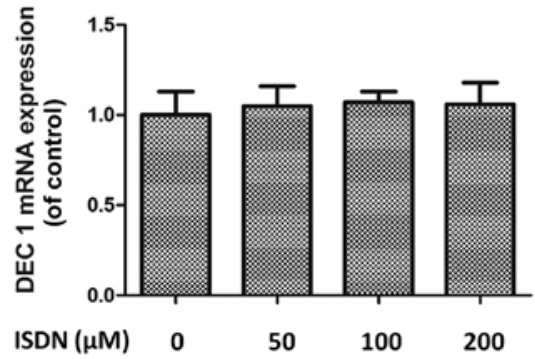

Figure 2. Isosorbide dinitrate (ISDN) does not have a notable effect on mesenchymal stem cell (MSCs) senescence. MSCs were cultured with ISDN at ISDN concentrations of 0 to $200 \mu \mathrm{M}$, and cellular senescence was compared between the groups. (A and B) p21 expression in MSCs was examined by western blot analysis; representative bands and a histogram are shown. The data are presented as the means \pm standard error of the mean (SEM); $\mathrm{n}=5$ in each group (C and D) DNA methyltransferase 1 DNMT1 mRNA expression and differentiated embryo chondrocyte expressed gene 1 (DEC1) mRNA expression in MSCs was analyzed by quantitative PCR; the results are shown in the bar diagram. Data were obtained from 6 independent experiments, presented as the means \pm SE, and compared with the control (untreated group; 0).

A

$$
\text { p21 }
$$

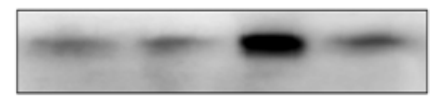

$\beta$-actin

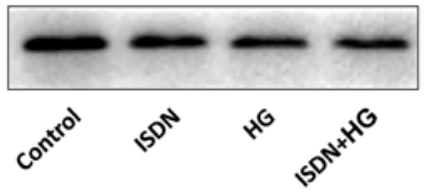

C

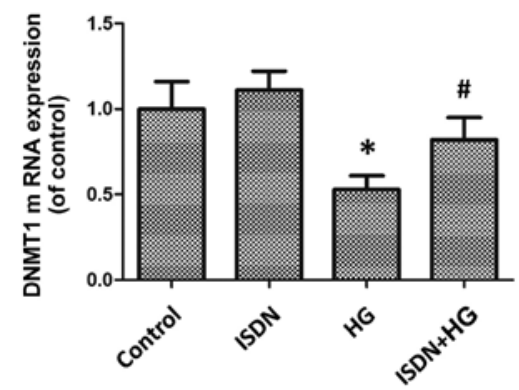

B

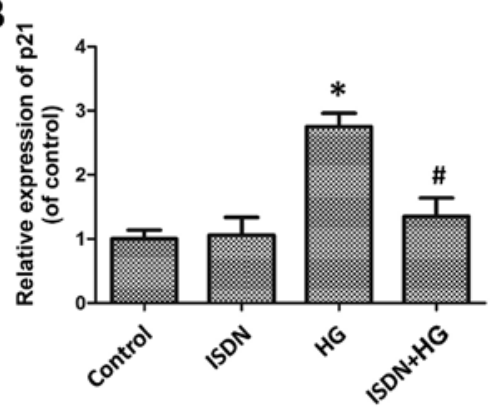

D

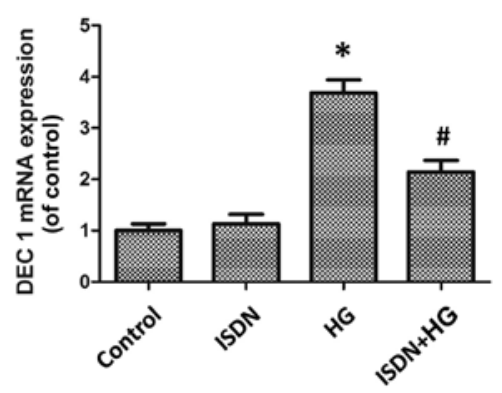

Figure 3. Isosorbide dinitrate (ISDN) preconditioning significantly attenuated the senescence of mesenchymal stem cells (MSCs) induced by high glucose (HG). MSCs were conditioned with or without ISDN $(50 \mu \mathrm{M})$ for $6 \mathrm{~h}$ prior to HG treatment $(33 \mathrm{mM})$ for an additional $24 \mathrm{~h}$; cellular senescence was then investigated between the groups. (A and B) Cell aging was measured based on p21 expression, which was assessed by western blot analysis, and representative bands and a statistical histogram are shown. The results are presented as the means \pm standard error of the mean (SEM); $n=4$ in each group. (C and D) Quantitative PCR was used to assess the mRNA expression of DNA methyltransferase 1 (DNMT1) and differentiated embryo chondrocyte expressed gene 1 (DEC1) in MSCs. The data are presented as the means $\pm \mathrm{SE}$ and are shown in the histogram. Data were confirmed in 5 independent experiments. ${ }^{*} \mathrm{P}<0.05$ vs. control; ${ }^{~} \mathrm{P}<0.05$ vs. the HG group.

ISDN treatment reduces $H G$-induced MSC senescence. To observe the effect of ISDN pre-treatment on HG-induced
MSC senescence, the MSCs were first cultured with ISDN at a concentration of 50,100 or $200 \mu \mathrm{M}$ for $24 \mathrm{~h}$ to determine 
A

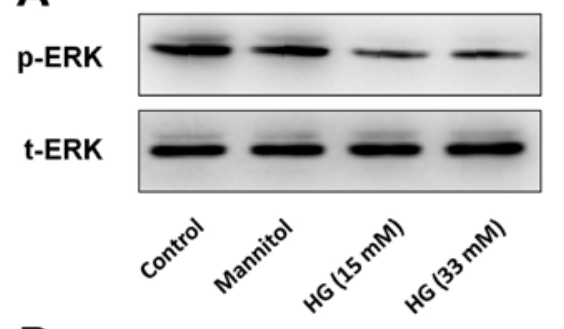

B

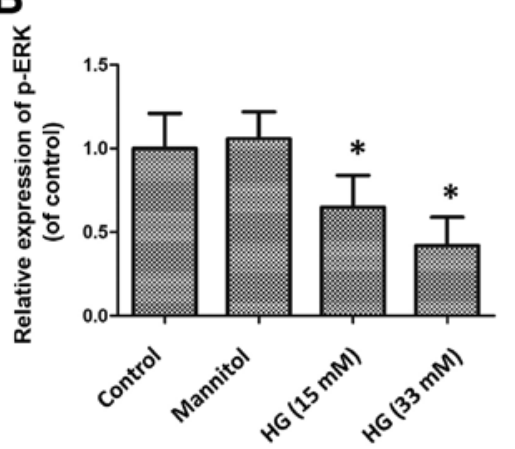

E

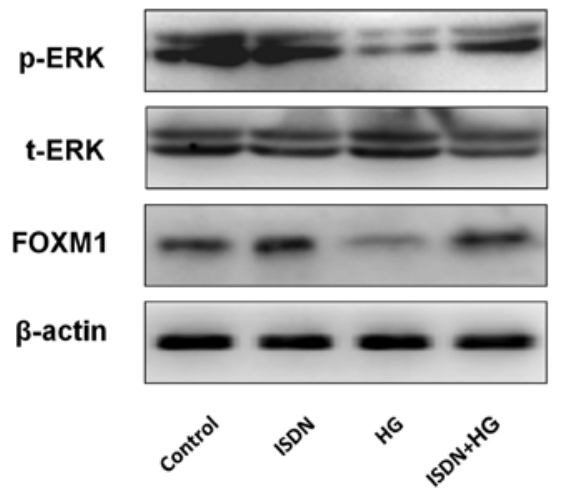

C

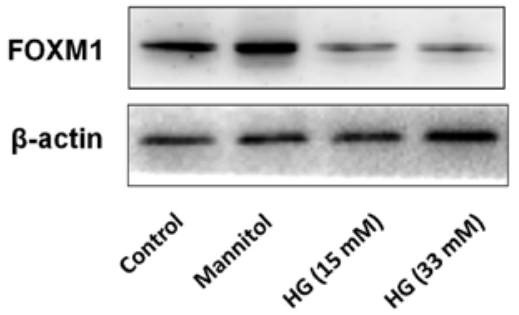

D
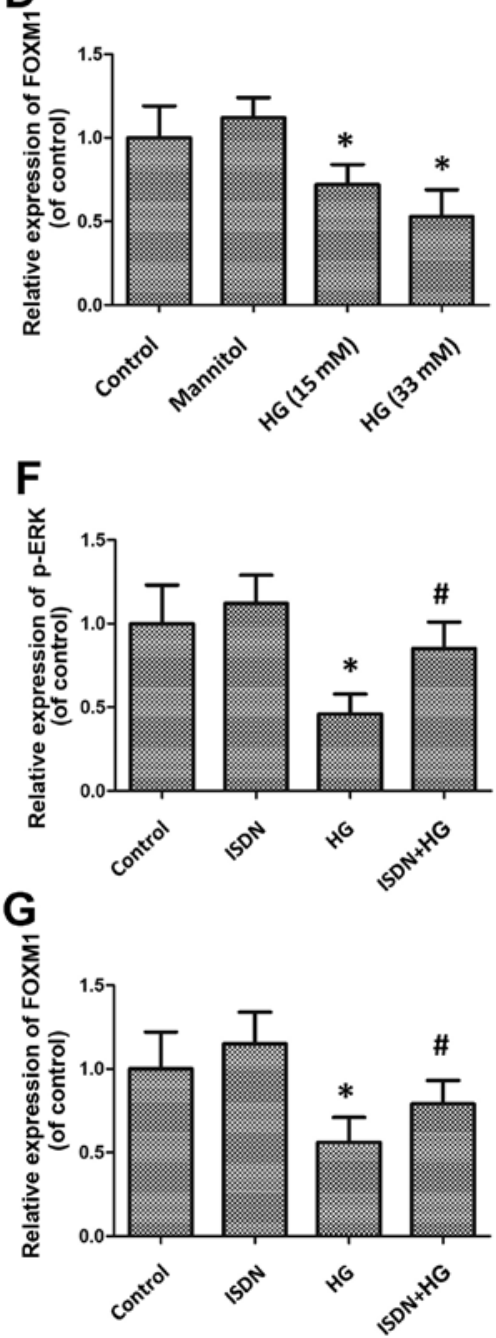

Figure 4. The ERK/FOXM1 pathway is affected by the induction of mesenchymal stem cell (MSC) senescence induced by high glucose (HG) and the protective effects of isosorbide dinitrate (ISDN). (A-D) MSCs were treated with HG at $15 \mathrm{mM}$ or $33 \mathrm{mM}$ or with mannitol (33 mM), which served as a negative control with an osmotic pressure equal to that of $33 \mathrm{mM} \mathrm{HG}$. ERK phosphorylation and FOXM1 expression were evaluated by western blot, and representative bands are shown. The results are shown as the means \pm standard error of the mean (SEM); $\mathrm{n}=3$ per group. * $\mathrm{P}<0.05$ vs. the control. (E-G) MSCs were preconditioned with ISDN $(50 \mu \mathrm{M})$ for $6 \mathrm{~h}$, followed by HG treatment $(33 \mathrm{mM})$ for an additional $24 \mathrm{~h}$. Western blot analysis was used to measure the activity of the ERK/ FOXM1 pathway: representative bands and a statistical bar diagram are shown. The data are presented as the means $\pm \mathrm{SEM} ; \mathrm{n}=3$ in each experiment. $\mathrm{P}<0.05$ vs. the control group; ${ }^{\mathrm{P}}<0.05$ vs. the $\mathrm{HG}$ group.

the appropriate intervention dose of ISDN. At these concentrations, ISDN did not exert a significant effect on MSC senescence compared with the control group, as evidenced by p21 expression and DNMT1 and DEC1 mRNA expression (all P>0.05) (Fig. 2).

Subsequently, the MSCs were incubated with $\operatorname{ISDN}(50 \mu \mathrm{M})$ for $6 \mathrm{~h}$ and then cultured with $33 \mathrm{mM} \mathrm{HG}$ for an additional $24 \mathrm{~h}$. Western blot analysis demonstrated that although incubation with $50 \mu \mathrm{M}$ ISDN did not affect p21 expression [ISDN group vs. control (untreated) group, $1.06 \pm 0.28$ vs. $1.00 \pm 0.14$;
$\mathrm{P}>0.05]$, treatment with $33 \mathrm{mM}$ HG significantly upregulated p21 expression in the MSCs [HG group vs. control (untreated) group, $2.75 \pm 0.21$ vs. $1.00 \pm 0.14 ; \mathrm{P}<0.05$ ], which was markedly attenuated after pre-conditioning with ISDN (ISDN + HG group vs. HG group, $1.35 \pm 0.29$ vs. $2.75 \pm 0.21$; $\mathrm{P}<0.05$ ) (Fig. $3 \mathrm{~A}$ and B). DNMT1 mRNA expression was significantly increased in the ISDN + HG group compared to the HG group $(0.82 \pm 0.13$ vs. $0.53 \pm 0.08, \mathrm{P}<0.05$ ) (Fig. $3 \mathrm{C}$ ); DEC1 mRNA expression was decreased in the ISDN + HG group compared to the HG group $(2.14 \pm 0.23$ vs. $3.68 \pm 0.26, \mathrm{P}<0.05)$ (Fig. $3 \mathrm{D})$. 
A
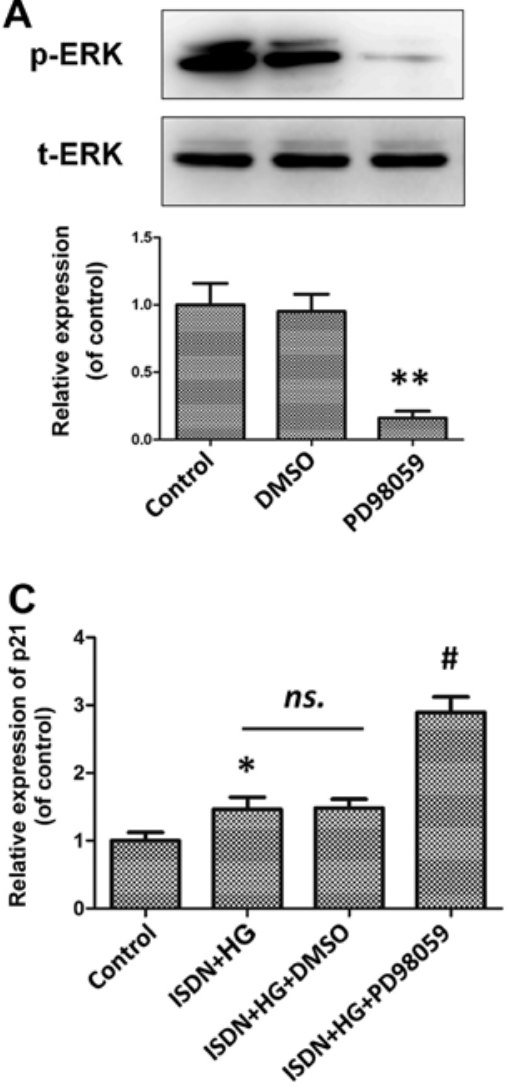
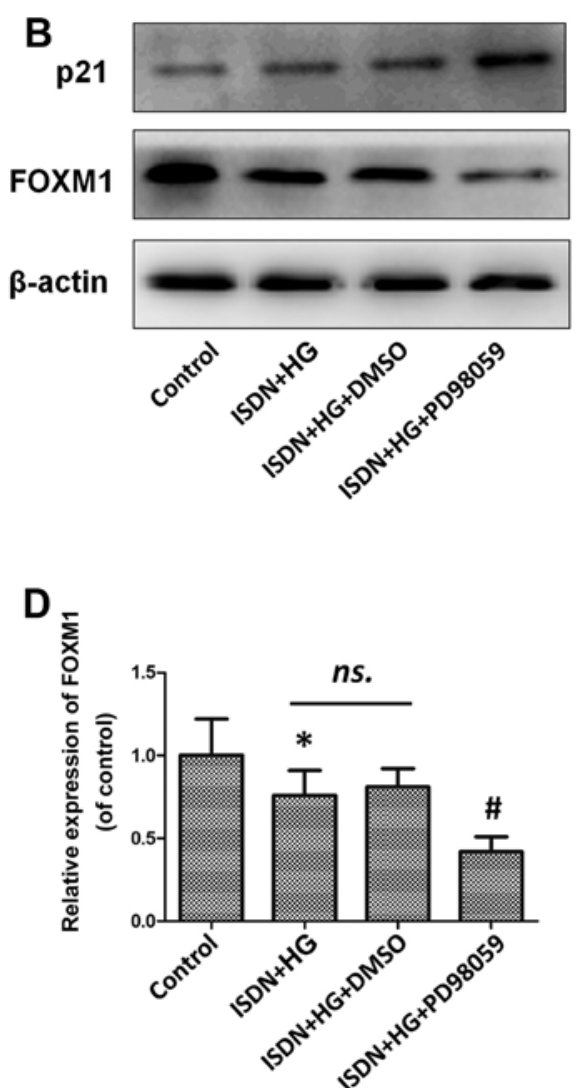

Figure 5. Inhibition of ERK phosphorylation abolishes the favourable effects of isosorbide dinitrate (ISDN) on mesenchymal stem cell (MSC) senescence. (A) MSCs were treated with PD98059 (ERK inhibitor; $20 \mu \mathrm{M}$ ) or DMSO (served as negative control) for 30 min, and ERK phosphorylation was then investigated by western blot analysis. Representative bands and a corresponding bar diagram are shown. ${ }^{* *} \mathrm{P}<0.01$ vs. the control group. (B-D) MSCs were incubated with PD98059 (20 $\left.\mu \mathrm{M}\right)$ or DMSO (served as negative control) for $30 \mathrm{~min}$, followed by ISDN pre-culture for $6 \mathrm{~h}$ and high glucose (HG) treatment for an additional $24 \mathrm{~h}$. Representative p21 and FOXM1 expression as determined by western blot analysis is shown, and the results are presented in the histogram. The data are presented as the means \pm standard error of the mean (SEM); $\mathrm{n}=3$ in each experiment. ${ }^{*} \mathrm{P}<0.05$ vs. the control group; ${ }^{*} \mathrm{P}<0.05$ vs. the ISDN + HG group; ns., not significant.

The ERK/FOXM1 pathway is involved in the protective effects of ISDN against MSC senescence. The ERK/FOXM1 pathway plays a key role in cellular senescence (19), and thus we investigated changes occurring in this pathway in MSCs following treatment with HG with or without pre-incubation with ISDN. HG induced the downregulation of ERK phosphorylation and FOXM1 expression in a dose-dependent manner (all $\mathrm{P}<0.05$ ) (Fig. 4A-D). At a concentration of $33 \mathrm{mM}, \mathrm{HG}$ decreased ERK phosphorylation and FOXM1 expression by 53.8 and $43.9 \%$, respectively, compared with the control (untreated) group (both $\mathrm{P}<0.05$ ); however, the suppressive effects on the ERK/ FOXM1 pathway were markedly reversed by pre-incubation with ISDN (ERK phosphorylation: ISDN + HG group vs. HG group, $0.85 \pm 0.16$ vs. $0.46 \pm 0.12, \mathrm{P}<0.05$; FOXM1 expression: ISDN + HG group vs. HG group, $0.79 \pm 0.14$ vs. $0.56 \pm 0.15$, $\mathrm{P}<0.05$ ) (Fig. 4E-G).

Activation of the ERK/FOXM1 pathway mediates the effects of ISDN on MSC senescence. To determine the role of the ERK/FOXM1 pathway in the effects of ISDN on HG-induced MSC senescence, PD98059, a specific inhibitor of the ERK pathway, and siRNA were used to inhibit the activity of the ERK/ FOXM1 pathway (Figs. 5A and 6A). PD98059 effectively inhibited ERK phosphorylation by $83.6 \%$ compared with the control (untreated) group $(\mathrm{P}<0.01)$, which significantly inhibited the beneficial effects of ISDN on MSC senescence, as indicated by the upregulation of $\mathrm{p} 21$ expression in the ISDN + HG + PD98059 group compared with the ISDN $+\mathrm{HG}$ group $(2.89 \pm 0.23$ vs. $1.46 \pm 0.18, \mathrm{P}<0.05$ ) (Fig. 5B and C). Similarly, following the knockdown of FOXM1 in the MSCs by $74.8 \%$ using RNA interference, the protective effects of ISDN on HG-induced MSC senescence were significantly attenuated, as indicated by a comparison of the ISDN + HG + siFOXM1 and ISDN + HG groups; there was an increase in $\mathrm{p} 21$ expression in the ISDN $+\mathrm{HG}+$ siFOXM1 group compared with the ISDN + HG group $(3.05 \pm 0.22$ vs. $1.44 \pm 0.24$, $\mathrm{P}<0.05$ ) (Fig. 6B and C) .

A senescence-associated SA- $\beta$-gal assay was performed to verify the beneficial effects of ISDN on MSC senescence as revealed above. A higher percentage of senescent MSCs with a typically flattened and enlarged cell shape accompanied by SA- $\beta$-gal positivity was observed in the $\mathrm{HG}$ group compared to the control group $(\mathrm{P}<0.05)$ (Fig. 7A and $\mathrm{B})$. Of note, preincubation with ISDN markedly decreased the HG-induced MSC senescence (ISDN + HG group vs. HG group, 10.9 \pm 3.72 vs. $32.8 \pm 6.83 \%$; $\mathrm{P}<0.05)$. However, treatment with PD98059 significantly attenuated the effects of ISDN on MSC aging, indicated by the increased number of SA- $\beta$-gal-positive MSCs $(29.4 \pm 5.81$ vs. $10.9 \pm 3.72 \%, \mathrm{P}<0.05)$ (Fig. $7 \mathrm{~A}$ and $\mathrm{B}$ ), as well as the decrease in DNMT1 mRNA expression $(0.45 \pm 0.05$ vs. $0.75 \pm 0.09, \mathrm{P}<0.05)($ Fig. $7 \mathrm{C})$, and the increase in DEC1 mRNA 

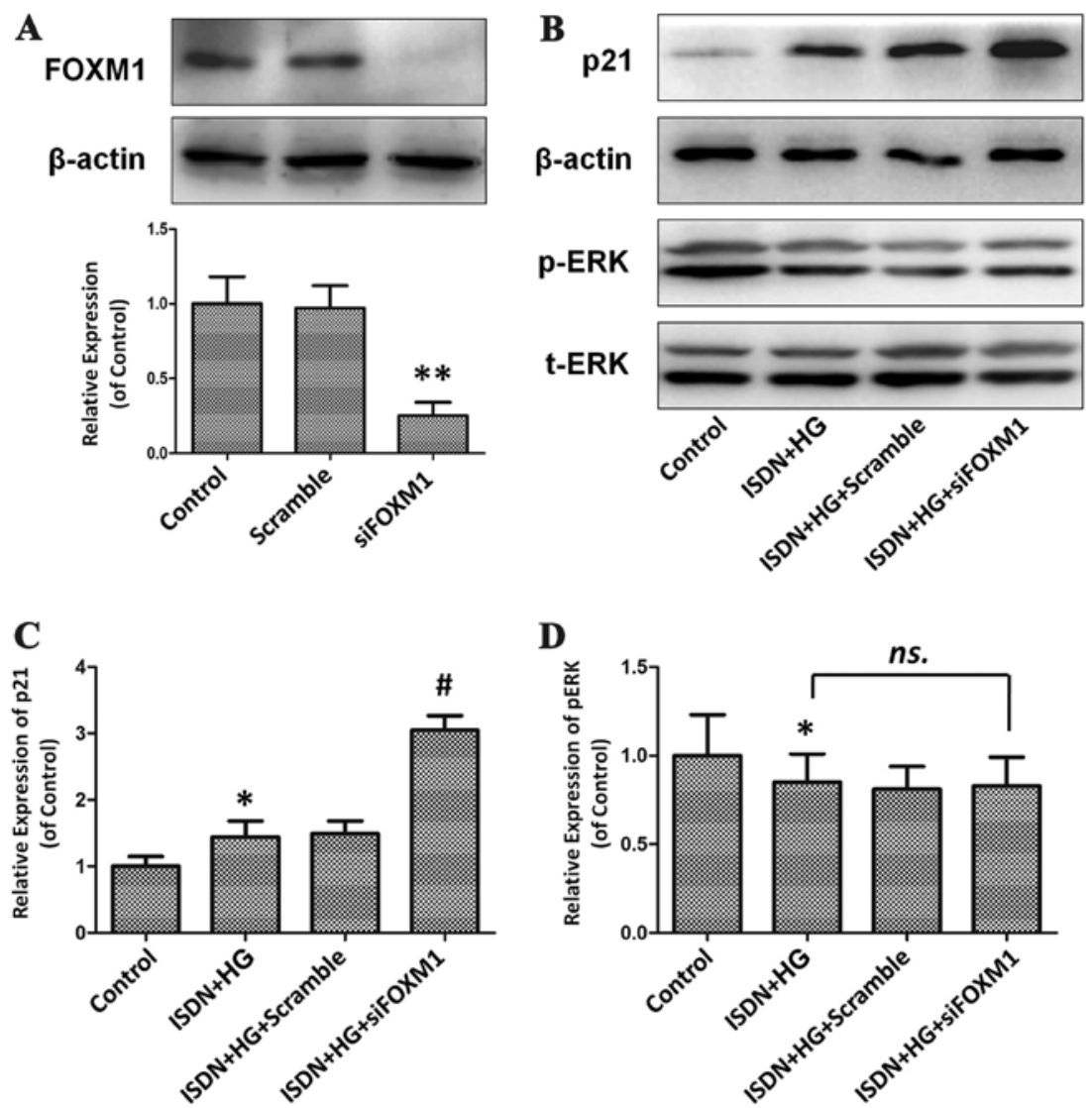

Figure 6. Knockdown of FOXM1 attenuates the beneficial effects of isosorbide dinitrate (ISDN) on mesenchymal stem cell (MSC) aging. (A) siRNA targeting FOXM1 or scrambled siRNA were transfected into the MSCs, and the representative bands and a histogram are shown with data presented as the means \pm standard error of the mean (SEM) and confirmed in 3 independent experiments. ${ }^{* *} \mathrm{P}<0.01$ vs. control group. (B-D) After the knockdown of FOXM1, MSCs were cultured with ISDN for $6 \mathrm{~h}$ and then treated with high glucose (HG) for another $24 \mathrm{~h}$. p21 expression and ERK phosphorylation levels were determined by western blot analysis and are statistically demonstrated in the histogram. Data are presented as the means $\pm \mathrm{SE}$ from 3 independent experiments. * $\mathrm{P}<0.05$ vs. the control group; ${ }^{\#} \mathrm{P}<0.05$ vs. the ISDN + HG group;.ns., not significant.

expression $(3.78 \pm 0.18$ vs. $2.26 \pm 0.21, \mathrm{P}<0.05)$ (Fig. $7 \mathrm{D})$ in the ISDN + HG + PD98059 group compared with the ISDN + HG group. Accordingly, FOXM1 knockdown markedly abolished the effects of ISDN in MSC senescence, which was indicated by the increase in the number of MSCs with SA- $\beta$-gal positivity $(28.3 \pm 5.25$ vs. $10.9 \pm 3.72 \%, \mathrm{P}<0.05)$ (Fig. $7 \mathrm{~A}$ and $\mathrm{B})$, as well as the decrease in DNMT1 mRNA expression $(0.42 \pm 0.08$ vs. $0.75 \pm 0.09, \mathrm{P}<0.05$ ) (Fig. 7C), and an increase in DEC1 mRNA expression $(3.64 \pm 0.24$ vs. $2.26 \pm 0.21, \mathrm{P}<0.05)$ (Fig. $7 \mathrm{D})$ in the $\mathrm{ISDN}+\mathrm{HG}+$ siFOXM1 group compared with the ISDN + HG group.

Upregulation of $\mathrm{miR}-130 \mathrm{~b}$ is associated with the activation of the ERK/FOXM1 pathway. The key regulatory role of the miR-130 family, including miR-130a and miR-130b, in cellular growth arrest has previously been demonstrated (14). In this study, we therefore examined the potential involvement of miR-130a and miR-130b in the response to HG treatment and the effects of ISDN. HG gradually suppressed miR-130b expression (all $\mathrm{P}<0.05$ ) in a concentration-dependent manner, but had no detectable effect on miR-130a expression (all $\mathrm{P}>0.05$ ) (Fig. 8A and B). Pre-treatment with ISDN reversed the decrease in miR-130b expression induced by HG stimulation (ISDN + HG group vs. HG group, $0.75 \pm 0.11$ vs. 0.39 \pm 0.07 ; $\mathrm{P}<0.05$ ), but had no apparent effect on miR-130a expression
(ISDN + HG group vs. HG group, $0.92 \pm 0.13$ vs. $0.93 \pm 0.11$; $\mathrm{P}>0.05$ ) (Fig. 8C and D). Furthermore, inhibition of the ERK/ FOXM1 pathway by either PD98059 or FOXM1 siRNA substantially abolished the recovery of miR-130b expression following pre-treatment with ISDN (ISDN + HG + PD98059 group vs. ISDN + HG group: $0.32 \pm 0.09$ vs. $0.75 \pm 0.11, \mathrm{P}<0.05$; ISDN + HG + siFOXM1 group vs. ISDN + HG group: $0.35 \pm 0.08$ vs. $0.75 \pm 0.11, \mathrm{P}<0.05$ ) (Fig. $8 \mathrm{E}$ and $\mathrm{F}$ ).

miR-130b is the key mediator of the effects of ISDN on MSC senescence. To determine whether miR-130b plays a key role in the protective effects of ISDN against MSC senescence, miR-130b was knocked down using antagomiR transfection and senescence-associated assays were then performed. It was found that after miR-130b expression was effectively downregulated by $74.3 \%$ compared to the control (Fig. 9A), the beneficial effects of ISDN on MSC senescence were markedly abolished, which was confirmed by the analysis of p21 expression $(1.47 \pm 0.29$ vs. $2.86 \pm 0.26, \mathrm{P}<0.05)$, DNMT1 mRNA expression $(0.72 \pm 0.13$ vs. $0.41 \pm 0.08, \mathrm{P}<0.05)$ and DEC1 mRNA expression $(2.39 \pm 0.28$ vs. $3.97 \pm 0.21, \mathrm{P}<0.05)$ (Fig. 9B-D) when comparing the ISDN + HG group with the ISDN + HG + antagomiR group.

ERK phosphorylation and FOXM1 activity were examined following the downregulation of miR-130b, indicating 
A

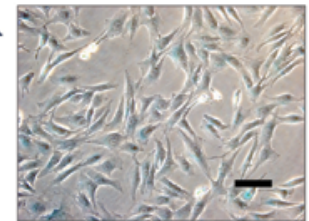

Control

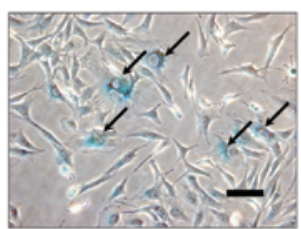

ISDN+HG+ PD98059

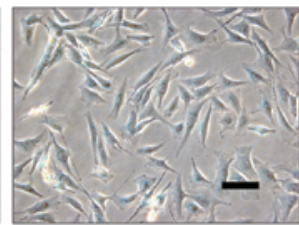

ISDN

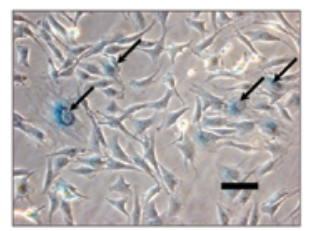

ISDN+HG+siFOXM1

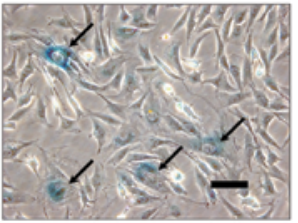

HG

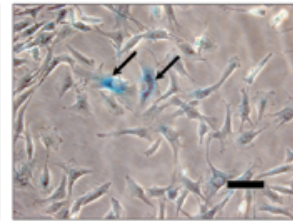

ISDN+HG
C

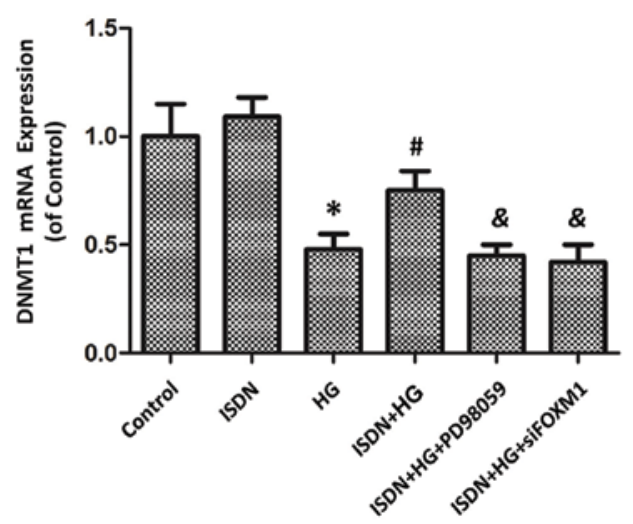

B

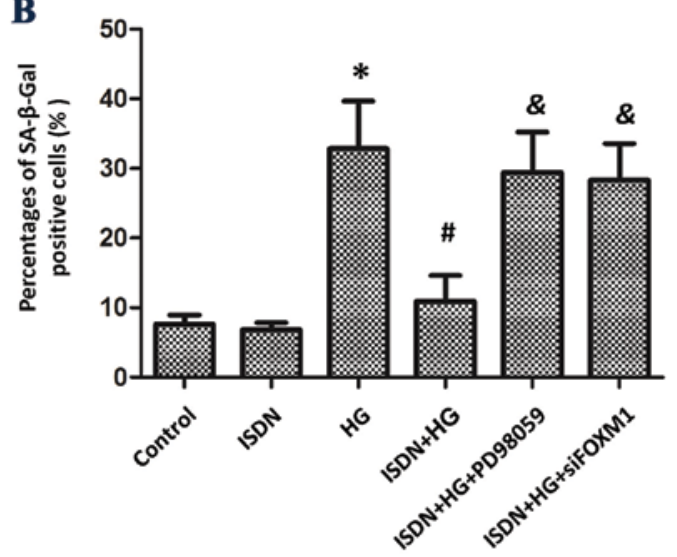

D

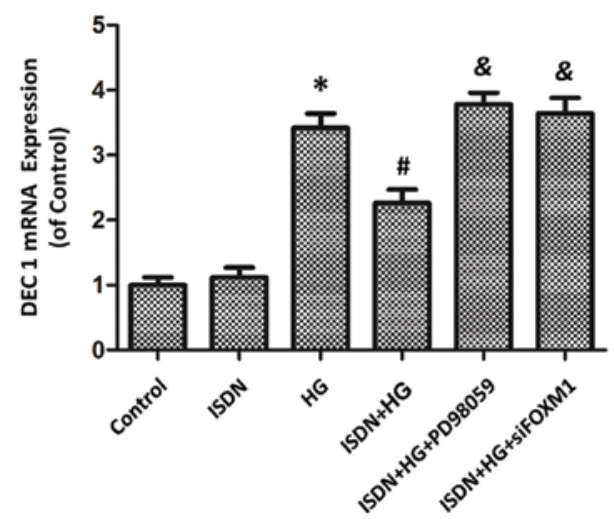

Figure 7. Isosorbide dinitrate (ISDN) pre-incubation delayed mesenchymal stem cell (MSC) senescence induced by high glucose (HG), which was mediated by the activation of the ERK/FOXM1 pathway. MSCs were incubated with PD98059 (ERK inhibitor; $20 \mu \mathrm{M}$ ) for 30 min prior to ISDN treatment (50 $\mu \mathrm{M}$ ) for $6 \mathrm{~h}$, followed by HG treatment $(33 \mathrm{mM})$ for an additional $24 \mathrm{~h}$. Alternatively, MSCs were treated with PD 98059 or subjected to FOXM1 knockdown by siRNA and then treated with ISDN and HG. Senescence-associated $\beta$-galactosidase (SA- $\beta$-gal) activity and differentiated embryo chondrocyte expressed gene 1 (DEC1) and DNA methyltransferase 1 (DNMT1) mRNA expression were assessed to determine the cellular aging in each group. (A and B) Representative photomicrograph of the SA- $\beta$-gal assay is shown (scale bar, $20 \mu \mathrm{m}$ ). The percentage of $\beta$-galactosidase-positive cells (indicated by arrows) in each group was compared and is illustrated in the histogram; $n=6$ in each experiment. (C and D) Quantitative PCR analysis revealing the differences in DEC1 and DNA methyltransferase 1 (DNMT1) mRNA expression in each group. The data are presented as the means \pm standard error of the mean (SEM); $\mathrm{n}=3$ in each experiment. "P<0.05 vs. the control group; ${ }^{*} \mathrm{P}<0.05$ vs. the HG group; ${ }^{\circledR} \mathrm{P}<0.05$ vs. the ISDN + HG group.

that there were no signficant changes between the control group and the antagomiR group, both in phosphorylated ERK $(1.00 \pm 0.16$ vs. $0.95 \pm 0.12, \mathrm{P}>0.05)$ and in FOXM1 expression $(1.00 \pm 0.13$ vs. $1.08 \pm 0.16, \mathrm{P}>0.05)$ (Fig. $9 \mathrm{E}$ and $\mathrm{F}$ ).

\section{Discussion}

This study aimed to investigate the effects of ISDN on HG-induced MSC senescence, demonstrating that ISDN preconditioning decreases SA- $\beta$-Gal activity in MSCs, decreases the expression of p21 protein and DEC1 mRNA, and increases the expression of DNMT1 mRNA, consistent with our original assumption that ISDN inhibits MSC aging triggered by HG. The activation of the ERK/FOXM1 pathway plays a pivotal role in this pathophysiological process, as evidenced by the attenuation of the favourable effects of ISDN by either the inhibition of ERK phosphorylation or the knockdown of FOXM1 in the MSCs. As a senescence-associated modulator, miRNA-130b was identified to function downstream of the ERK/FOXM1 pathway, collectively contributing to the protective effects of ISDN against MSC aging.

Diabetic patients frequently suffer from micro- or macrovascular abnormalities, including neuropathy, nephropathy, retinopathy and accelerated ischemic heart diseases, which 
$\mathbf{A}$
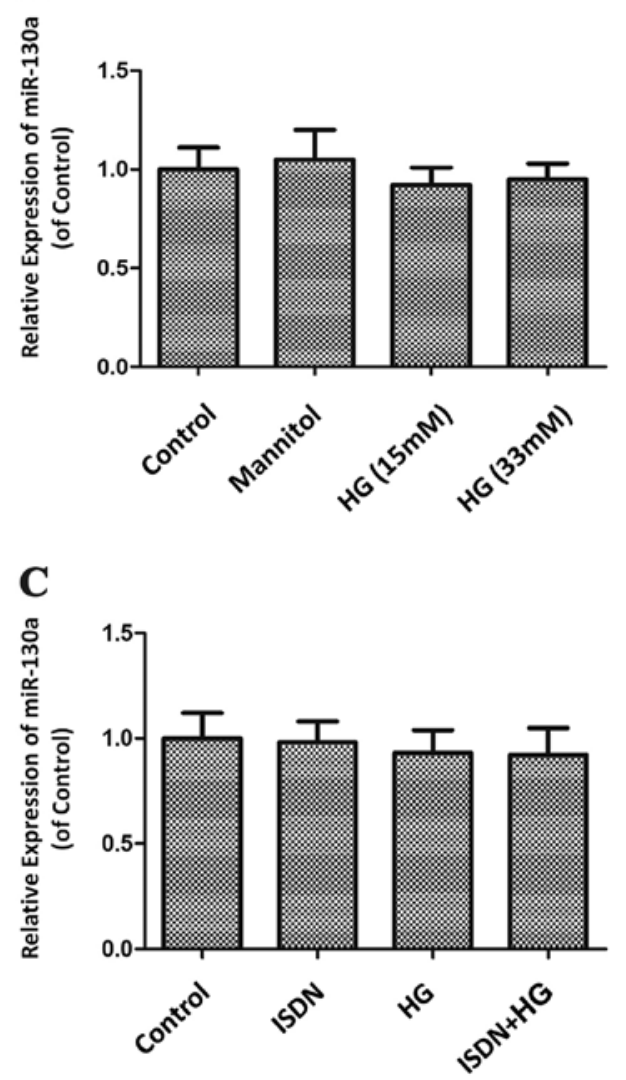

$\mathbf{E}$

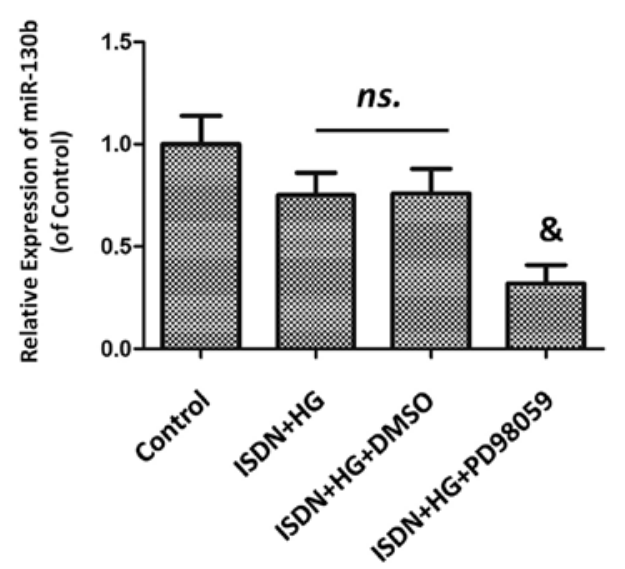

B

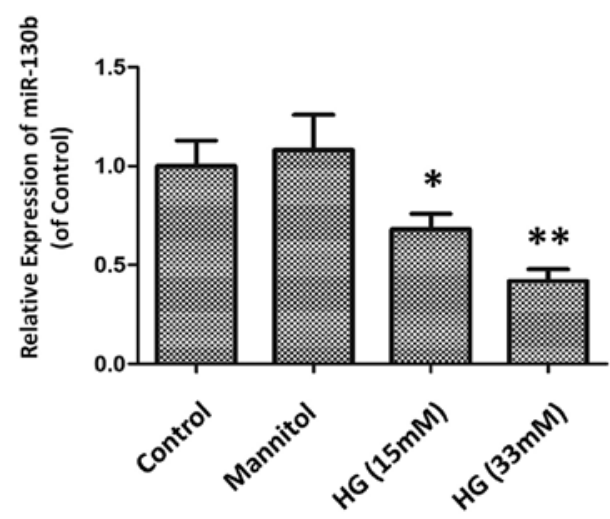

D

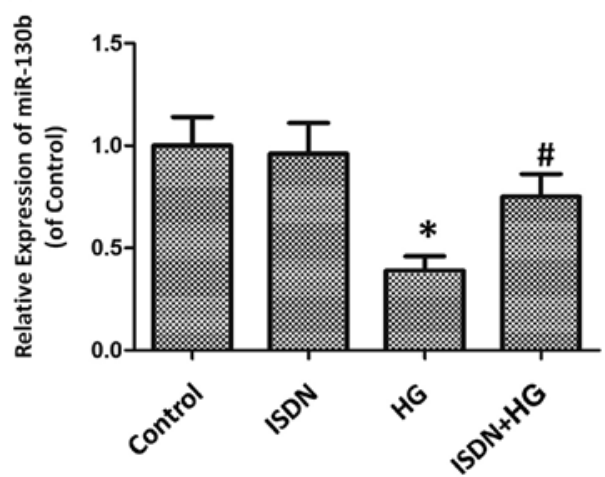

$\mathbf{F}$

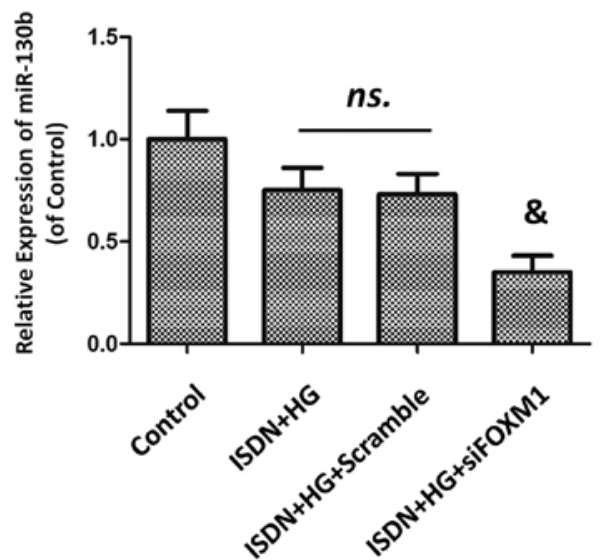

Figure 8. miR-130b, not miR-130a, is upregulated by the activation of the ERK/FOXM1 cascade during the protection of high glucose (HG)-induced mesenchymal stem cell (MSC) senescence by isosorbide dinitrate (ISDN). (A and B) MSCs were exposed to HG at progressively increasing concentrations for $24 \mathrm{~h}$ or to mannitol $(33 \mathrm{mM})$, which served as a negative control with an osmotic pressure equal to that of $33 \mathrm{mM} \mathrm{HG}$. The expression of the miR-130 family, including miR-130a and $-130 \mathrm{~b}$, was determined by quantitative PCR and is shown in the histogram. The data are presented as the means \pm standard error of the mean (SEM); $\mathrm{n}=3$ in each experiment. ${ }^{*} \mathrm{P}<0.05,{ }^{* *} \mathrm{P}<0.01$ vs. the control group. (C and D) MSCs were incubated with or without ISDN $(50 \mu \mathrm{M})$ for $6 \mathrm{~h}$, followed by HG treatment ( $\left.33 \mathrm{mM}\right)$ for an additional $24 \mathrm{~h}$. The expression of miR-130a/b was analyzed by quantitative PCR, and the results are presented in the histogram. The data are presented as the means \pm standard error of the mean (SEM); $n=3$ in each experiment. ${ }^{*} \mathrm{P}<0.05$ vs. the control group; ${ }^{*} \mathrm{P}<0.05$ vs. the HG group. (E and F) MSCs were cultured with PD98059 $(20 \mu \mathrm{M})$ for $30 \mathrm{~min}$ prior to ISDN treatment $(50 \mu \mathrm{M}, 6 \mathrm{~h})$, followed by HG treatment (33 mM, $24 \mathrm{~h})$. Alternatively, MSCs were treated with PD98059 or subjected to FOXM1 knockdown by siRNA and then treated with ISDN and HG. miR-130b expression was evaluated by quantitative PCR, and the results are shown in the bar diagram. The data are presented as the means \pm SEM; $n=3$ in each experiment. ns., not significant; ${ }^{\&} \mathrm{P}<0.05 \mathrm{vs.}$ the ISDN $+\mathrm{HG}$ group.

mobilize MSCs to repair and regenerate damaged tissue. Although there is a complex condition which induces MSC growth arrest, it is undoubtedly considered that HG is the primary trigger. Accordingly, HG incubation, not oxidative stress or ultraviolet B radiation (UVB) treatment, was developed to establish a cellular senescence model in MSCs, which may better simulate the true pathophysiological state in diabetes mellitus (DM) (20), and may have great practical implications for the treatment of patients with DM accompanied by its various complications.

Organic nitrates are one of the most common types of drugs used for the long-term treatment of acute coronary syndrome 
$\mathbf{A}$

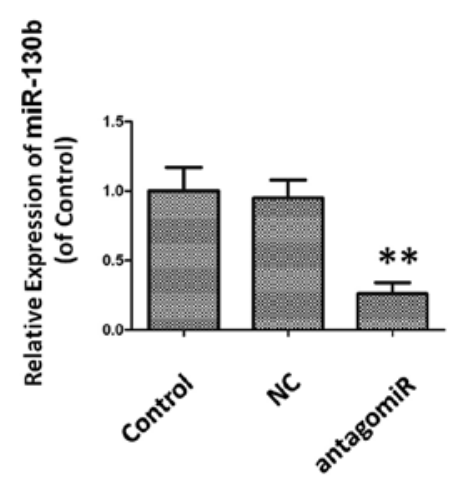

C
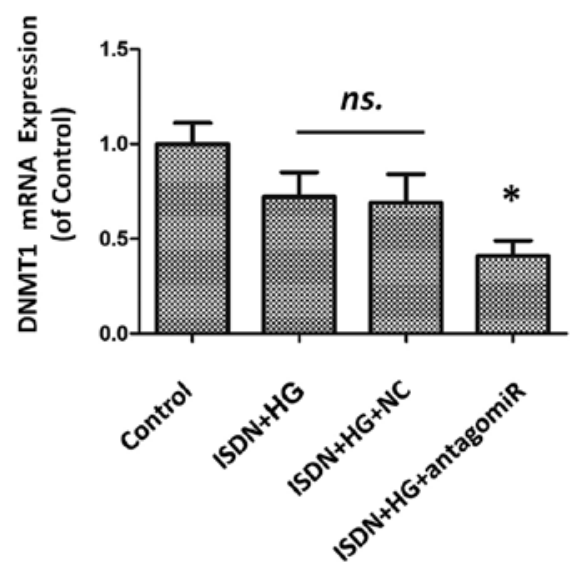

$\mathbf{E}$
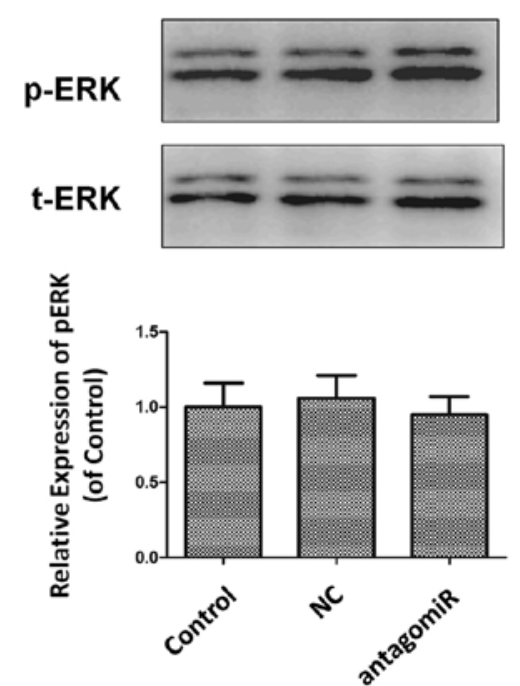

B $\quad$ 21

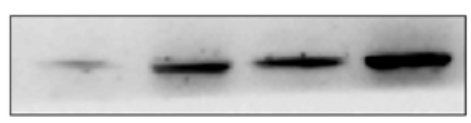

$\beta$-actin
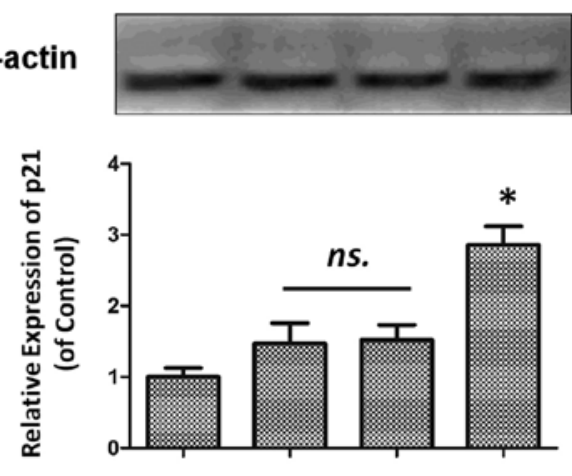

D
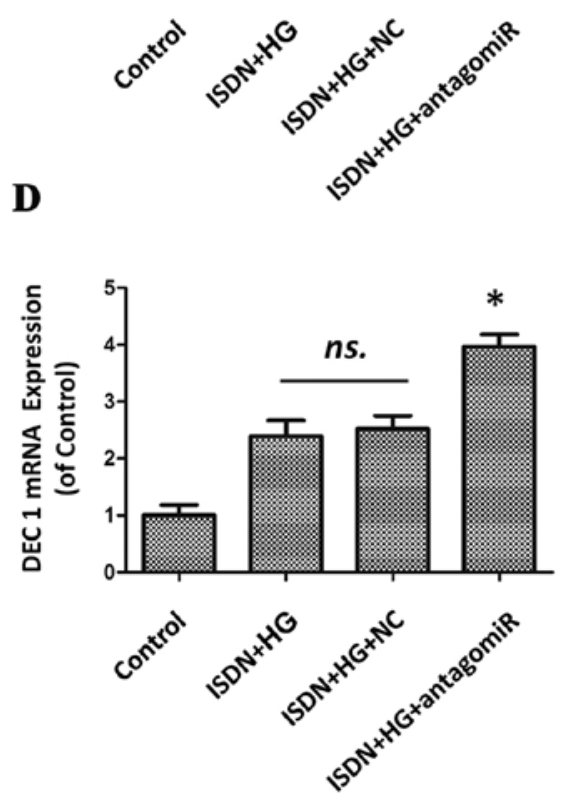

$\mathbf{F}$
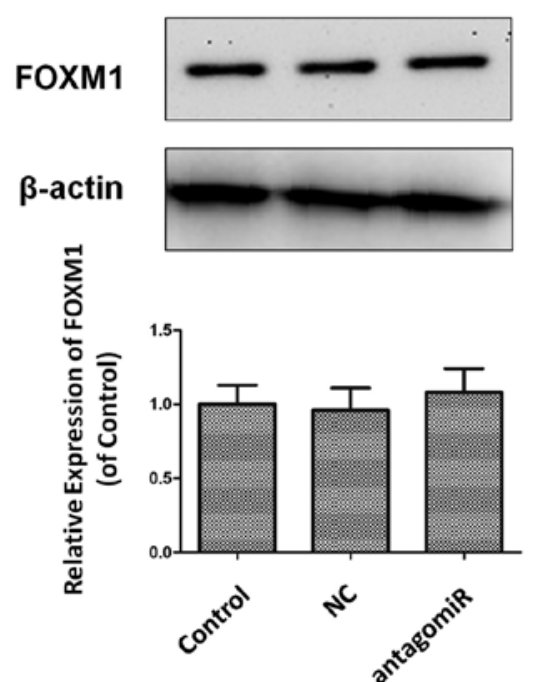

Figure 9. Downregulation of miR-130b abolishes the beneficial effects of isosorbide dinitrate (ISDN) on mesenchymal stem cell (MSC) aging. (A) The miR$130 \mathrm{~b}$ level was quantified by quantitative PCR. The expression value of the control group was designated 1 , and the levels of other groups were calibrated to this value. Data was presented as the means \pm standard error of the mean (SEM) and confirmed in 5 independent experiments. ** P<0.01 vs. control group. (B-D) Follwing the downregulation of miR-130b, MSCs were incubated with ISDN for $6 \mathrm{~h}$ and then exposed to high glucose (HG) for an additional $24 \mathrm{~h}$. p21 expression was determined by western blot analysis. Quantitative PCR detection was further performed to reveal the differences in differentiated embryo chondrocyte expressed gene 1 (DEC1) and DNA methyltransferase 1 (DNMT1) mRNA expression in each group. The data are presented as the means \pm SEM from 5 independent experiments. ${ }^{*} \mathrm{P}<0.05$ vs. the ISDN + HG group; ns., not significant. (E-F) ERK phosphorylation and FOXM1 expression were determined following the suppression of miR-130b. Representative western blots are shown and the results are presented in the bar diagram as the means \pm SEM from 5 independent experiments. NC, negative control. 
and acute or chronic congestive heart failure, as well as coronary heart diseases (21). The release of NO is the main function of ISDN (22) and leads to the activation of soluble guanylate cyclase and the reduction of the intracellular calcium content, subsequently resulting in the relaxation of target cells, primarily the vascular smooth muscle (23). However, a comprehensive understanding of the mechanisms of action of organic nitrates, particularly other non-hemodynamic effects on other various cells, has not yet been established (24). Given the critical role of MSCs in cardiovascular diseases, we wished to determine whether treatment with ISDN affects the biological characteristics of MSCs.

Senescent MSCs exhibit a significantly reduced ability to self-renew, growth is terminated at an early stage at approximately 40-50 population doublings, and a decline in differentiation potential and proliferation rate is observed (25). Furthermore, senescent MSCs provide a weak protective effect due to the decreased activity of repair and antioxidant enzymes in aged MSCs, resulting in the unsatisfactory effects of MSC-based treatments for tissue repair (26). Several pathophysiological states, such as HG and oxidative stress, are considered critical contributors to cell senescence, as well as predominant risks or accompanying factors in cardiac ischemia which is regularly treated with organic nitrates (27). Therefore, our identification of ISDN as a modulator of MSC senescence may provide insight into the protective mechanisms of organic nitrates in cardiovascular diseases. However, an investigation using DM models in vivo, comparing MSC senescence before and after receiving organic nitrate treatment, is required in order to further confirm the in vitro above findings.

The ERK/FOXM1 pathway is involved in cell cycle regulation and senescence modulation $(12,13)$. However, whether it accelerates or delays cell aging is dependent on the cell type and pathophysiological conditions. Ling et al (28) observed that the activated ERK phosphorylation and upregulated FOXM1 expression were essential for the protective effects of low-power laser irradiation against NIH3T3 cell senescence induced by UVB. By contrast, Wang et al (19) demonstrated that the $\mathrm{N}$ terminus of ASPP2 binds to Ras, enhancing Ras/ Raf/MEK/ERK activation to facilitate oncogene-induced senescence; Zeng et al (29) discovered that FOXM1 is upregulated in gastric cancer and that its inhibition led to termination of the cell cycle at the G0/G1 phase, partially dependent on p27 kip1. In light of these results, we examined changes in the activities of ERK and FOXM1 in HG-treated MSCs and in response to the protective effects of ISDN. HG significantly suppressed ERK phosphorylation and FOXM1 expression, which were both attenuated by ISDN preconditioning. Furthermore, the inhibition of ERK phosphorylation or the knockdown of FOXM1 attenuated the favourable effects of ISDN on MSC aging, indicating that ERK and FOXM1 are key mediators of the effects of ISDN. While the inhibition of ERK phosphorylation markedly decreased the upregulation of FOXM1, the downregulation of FOXM1 did not exert a significant effect on ERK activity, revealing the regulatory association between these two proteins.

Considering the crucial function of miRNAs in cell biology, we also investigated the senescence-associated miRNAs based on the review by Abdelmohsen et al (14), including those that elicit their actions through the pRB/p16 pathway (miR-106,
miR-130 and miR-24) or the p53 pathway (miR-34), affect senescence-associated secretory phenotypes (SASP) (miR-146) and modulate other senescence regulatory proteins (miR-29, miR-30 and miR-519). As demonstrated by quantitative PCR (data not shown), we determined that only miR-130b and not miR-130a is involved in HG-induced MSC aging, as well as the subsequent ISDN protective effects. Subsequently, we examined miR-130b expression following the suppression of the ERK/ FOXM1 cascade and hence deduced that miR-130b functions downstream of this pathway. Based on previous studies, it has been demonstrated that miR-130b inhibits the expression of p21, which is regarded to be antagonistic to p53/p21 pathway activity, leading to a recovery of growth arrest in proliferative cells $(30,31)$. Borgdorff et al (32) established an important role for the cyclin-dependent kinase (CDK) inhibitor, p21 (Waf1/Cip1), in the growth control of human mammary epithelial cells (HMECs) and analyzed the repertoire of miRNAs that modulate the activity of this tumor suppressor, demonstrating that miR-130b reduced p21 expression and in turn blocked Ras (G12V)-induced senescence. They further revealed that the overexpression of miR-130b rescued HMECs from Ras-induced senescence through the prevention of Ras-induced upregulation of p21, verifying the key role of miR-130b during proliferative cell senescence, which powerfully supported the underlying mechanisms found in our study. Considering that the regulation of miRNAs differs depending on cell type, we wished to determine whether the inhibition of miR-130b reduces the protective effects of ISDN on MSC senescence. We therefore silenced miR-130b expression in MSCs, revealing that miR$130 \mathrm{~b}$ downregulation markedly attenuated the advantageous effects of ISDN on MSC senescence, which was indicated by the detection of p21 expression and the mRNA expression of DNMT1 and DEC1. Furthermore, we revealed that both ERK phosphorylation and FOXM1 expression were not significantly affected by miR-130b suppression, together with our prior finding that the suppression of either ERK phosphorylation or FOXM1 expression markedly reduced miR-130b expression, collectively indicating that miR-130b resides downstream of the ERK/FOXM1 pathway. However, the exploration of the role of miR-130b in MSC senescence in the current study is preliminary, and further research focusing on the presumed target of miR-130b is required, including the prediction of informatics algorithm, luciferase report assay and functional complementary experiments. We aim to resolve these issues in our future research, which may broaden our understanding of the association between MSC senescence and miRNA modulation.

Support for our hypothesis can also be found in previous studies of the pharmacological function of organic nitrates on cell senescence or growth arrest. Huang et al (33) demonstrated that nitroglycerine (NTG) within a concentration range of 0.1-10 $\mu \mathrm{mol} / 1$ notably increased BrdU incorporation into human MSCs in a dose-dependent manner, suggesting that NTG enhances the cell growth rate and activity. The authors further determined that the promotion of NO release in MSCs by NTG was a key mechanism underlying this effect, suggesting that organic nitrate stimulates NO production in MSCs, leading to the increased cell viability. Moreover, the use of organic nitrates in phytophysiology (34) has indicated that nitrate limitation or starvation markedly induces leaf senescence based on a combined ${ }^{15} \mathrm{~N}$ tracing/proteomics 
analysis (35) and SAG12-transgenic plant construction (36). In addition, it has been reported that the long-term inhibition of NO synthase by L-arginine analogues, such as N( $\omega)$-nitrol-arginine methyl ester (L-NAME), accelerates vascular senescence in vitro and systemic hypertension and arteriosclerosis in vivo (37). Furthermore, it has been demonstrated that pre-treatment with various Chinese traditional medicines promotes the renovation of $\mathrm{H}_{2} \mathrm{O}_{2}$-stimulated senescence in human umbilical endothelial cells via nitrate production (38); and that elevated NO production, which can also be promoted by organic nitrates, in MSCs overexpressing eNOS enhances cellular activity and migration through the upregulation of stromal cell-derived factor-1 $\alpha$ (39), which conformably evidenced the significance and reasonability of our novel finding.

In conclusion, to the best of our knowledge, the present study provides evidence that ISDN exerts protective effects agaisnt HG-induced MSC senescence, and that the underlying mechanisms involve the activation of the ERK/FOXM1 pathway and the upregulation of miR-130b. The data presented in this study may prove to be of clinical value for patients with DM accompanied by diverse clinical complications.

\section{Acknowledgements}

This study was supported by grants from the National Natural Science Foundation of China (project nos. 81100145, $81370003,81300082,81470467$ and 81370322), the National Science Foundation for Post-doctoral Scientists of China (project nos. 2013M531124 and 2014T70391), the Medical Guiding Foundation of Science and Technology Commission of Shanghai (project no. 124119a7701), the Chinese Medical Doctor Association Foundation for Cardiovascular Research (project nos. DFCMDA201259 ands DFCMDA201255), the Key Specialty Construction of Medical Program in Shanghai (ZK2012A24) and the National Basic Research Program of China (973 Program, project no. 2012CB518605).

\section{References}

1. Sung YH, Shin MS, Ko IG, et al: Ulinastatin suppresses lipopolysaccharide-induced prostaglandin E-2 synthesis and nitric oxide production through the downregulation of nuclear factor- $\kappa \mathrm{B}$ in BV2 mouse microglial cells. Int J Mol Med 31: 1030-1036, 2013.

2. Navascues J, Perez-Rontome C, Gay M, et al: Leghemoglobin green derivatives with nitrated hemes evidence production of highly reactive nitrogen species during aging of legume nodules. Proc Natl Acad Sci USA 109: 2660-2665, 2012.

3. Matsui-Hirai H, Hayashi T, Yamamoto S, et al: Dose-dependent modulatory effects of insulin on glucose-induced endothelial senescence in vitro and in vivo: a relationship between telomeres and nitric oxide. J Pharmacol Exp Ther 337: 591-599, 2011.

4. Ransohoff JD and Wu JC: Imaging stem cell therapy for the treatment of peripheral arterial disease. Curr Vasc Pharmacol 10: 361-373, 2012

5. Xu J, Qian J, Xie X, et al: High density lipoprotein cholesterol promotes the proliferation of bone-derived mesenchymal stem cells via binding scavenger receptor-B type I and activation of PI3K/Akt, MAPK/ERK1/2 pathways. Mol Cell Biochem 371: 55-64, 2012.

6. Hou M, Liu J, Liu F, Liu K and Yu B: Clq tumor necrosis factor-related protein-3 protects mesenchymal stem cells against hypoxia- and serum deprivation-induced apoptosis through the phosphoinositide 3-kinase/Akt pathway. Int J Mol Med 33: 97-104, 2014.
7. Severino V, Alessio N, Farina A, et al: Insulin-like growth factor binding proteins 4 and 7 released by senescent cells promote premature senescence in mesenchymal stem cells. Cell Death Dis 4: e911, 2013.

8. Liu Y, Li Z, Liu T, et al: Impaired cardioprotective function of transplantation of mesenchymal stem cells from patients with diabetes mellitus to rats with experimentally induced myocardial infarction. Cardiovasc Diabetol 12: 40, 2013.

9. Lauer T, Heiss C, Balzer J, et al: Age-dependent endothelial dysfunction is associated with failure to increase plasma nitrite in response to exercise. Basic Res Cardiol 103: 291-297, 2008.

10. Ward MR, Connelly KA, Vijayaraghavan R, et al: eNOS overexpressing bone marrow cells are safe and effective in a porcine model of myocardial regeneration following acute myocardial infarction. Cardiovasc Ther 31: e72-e78, 2013.

11. Yang MY, Lin PM, Liu YC, et al: Induction of cellular senescence by doxorubicin is associated with upregulated miR-375 and induction of autophagy in K562 cells. PLoS One 7: e37205, 2012.

12. Bonet C, Giuliano S, Ohanna M, et al: Aurora B is regulated by the mitogen-activated protein kinase/extracellular signalregulated kinase (MAPK/ERK) signaling pathway and is a valuable potential target in melanoma cells. J Biol Chem 287: 29887-29898, 2012.

13. Qu K, Xu X, Liu C, et al: Negative regulation of transcription factor FoxM1 by p53 enhances oxaliplatin-induced senescence in hepatocellular carcinoma. Cancer Lett 331: 105-114, 2013.

14. Abdelmohsen K, Srikantan S, Kang MJ and Gorospe M: Regulation of senescence by microRNA biogenesis factors. Ageing Res Rev 11: 491-500, 2012.

15. Xu J, Qian J, Xie X, et al: High density lipoprotein protects mesenchymal stem cells from oxidative stress-induced apoptosis via activation of the PI3K/Akt pathway and suppression of reactive oxygen species. Int J Mol Sci 13: 17104-17120, 2012.

16. Wang Z, Ahmad A, Banerjee S, et al: FoxM1 is a novel target of a natural agent in pancreatic cancer. Pharm Res 27: 1159-1168, 2010.

17. Popovic R, Riesbeck LE, Velu CS, et al: Regulation of mir-196b by MLL and its overexpression by MLL fusions contributes to immortalization. Blood 113: 3314-3322, 2009.

18. Zhong W, Zou G, Gu J and Zhang J: L-arginine attenuates high glucose-accelerated senescence in human umbilical vein endothelial cells. Diabetes Res Clin Pract 89: 38-45, 2010.

19. Wang Z, Liu Y, Takahashi M, et al: $\mathrm{N}$ terminus of ASPP2 binds to Ras and enhances Ras/Raf/MEK/ERK activation to promote oncogene-induced senescence. Proc Natl Acad Sci USA 110: 312-317, 2013.

20. Yokoi T, Fukuo K, Yasuda O, et al: Apoptosis signal-regulating kinase 1 mediates cellular senescence induced by high glucose in endothelial cells. Diabetes 55: 1660-1665, 2006.

21. Munzel T: Recent studies on nitrates: their action, bioactivation and development of tolerance. Dtsch Med Wochenschr 133: 2277-2282, 2008 (In German).

22. Wang ZQ, Xu JF, Wang JP, Zhao WJ and Zeng M: Involvement of guanylate cyclase and $\mathrm{K}^{+}$channels in relaxation evoked by ferulate nitrate in rat aorta artery. J Pharmacol Sci 118: 521-530, 2012.

23. Thum T, Wiebking V, Ertl G and Bauersachs J: Organic nitrates differentially modulate circulating endothelial progenitor cells and endothelial function in patients with symptomatic coronary artery disease. Antioxid Redox Signal 15: 925-931, 2011.

24. Gori T and Daiber A: Non-hemodynamic effects of organic nitrates and the distinctive characteristics of pentaerithrityl tetranitrate. Am J Cardiovasc Drugs 9: 7-15, 2009.

25. Johnston PV,SasanoT, Mills K, et al: Engraftment, differentiation, and functional benefits of autologous cardiosphere-derived cells in porcine ischemic cardiomyopathy. Circulation 120: 1075-1083, 2009.

26. Williams AR and Hare JM: Mesenchymal stem cells: biology, pathophysiology, translational findings, and therapeutic implications for cardiac disease. Circ Res 109: 923-940, 2011.

27. Oelze M, Schuhmacher S and Daiber A: Organic nitrates and nitrate resistance in diabetes: the role of vascular dysfunction and oxidative stress with emphasis on antioxidant properties of pentaerithrityl tetranitrate. Exp Diabetes Res 2010: 213176, 2010.

28. Ling Q, Meng C, Chen Q and Xing D: Activated ERK/FOXM1 pathway by low-power laser irradiation inhibits UVB-induced senescence through down-regulating p21 expression. J Cell Physiol 229: 108-116, 2014.

29. Zeng J, Wang L, Li Q, et al: FoxM1 is up-regulated in gastric cancer and its inhibition leads to cellular senescence, partially dependent on p27 kip1. J Pathol 218: 419-427, 2009. 
30. Gorospe $\mathrm{M}$ and Abdelmohsen K: MicroRegulators come of age in senescence. Trends Genet 27: 233-241, 2011.

31. Su X, Chakravarti D, Cho MS, et al: TAp63 suppresses metastasis through coordinate regulation of Dicer and miRNAs. Nature 467: 986-990, 2010.

32. Borgdorff V, Lleonart ME, Bishop CL, et al: Multiple microRNAs rescue from Ras-induced senescence by inhibiting p21(Waf1/Cip1). Oncogene 29: 2262-2271, 2010.

33. Huang L, Qiu N, Zhang C, et al: Nitroglycerin enhances proliferation and osteoblastic differentiation in human mesenchymal stem cells via nitric oxide pathway. Acta Pharmacol Sin 29: 580-586, 2008

34. Guiboileau A, Yoshimoto K, Soulay F, Bataille MP, Avice JC and Masclaux-Daubresse C: Autophagy machinery controls nitrogen remobilization at the whole-plant level under both limiting and ample nitrate conditions in Arabidopsis. New Phytol 194: 732-740, 2012.

35. Desclos M, Etienne P, Coquet L, et al: A combined N15 tracing/ proteomics study in Brassica napus reveals the chronology of proteomics events associated with $\mathrm{N}$ remobilisation during leaf senescence induced by nitrate limitation or starvation. Proteomics 9: 3580-3608, 2009.
36. Sykorova B, Kuresova G, Daskalova S, et al: Senescence-induced ectopic expression of the A.tumefaciens ipt gene in wheat delays leaf senescence, increases cytokinin content, nitrate influx, and nitrate reductase activity, but does not affect grain yield. J Exp Bot 59: 377-387, 2008.

37. Boe AE, Eren M, Murphy SB, et al: Plasminogen activator inhibitor-1 antagonist TM5441 attenuates N $\omega$-nitro-L-arginine methyl ester-induced hypertension and vascular senescence. Circulation 128: 2318-2324, 2013.

38. Liu DH, Chen YM, Liu Y, et al: Ginsenoside Rb1 reverses $\mathrm{H}_{2} \mathrm{O}_{2}$-induced senescence in human umbilical endothelial cells: involvement of eNOS pathway J Cardiovasc Pharmacol 59: 222-230, 2012

39. Li N, Lu X, Zhao X, et al: Endothelial nitric oxide synthase promotes bone marrow stromal cell migration to the ischemic myocardium via upregulation of stromal cell-derived factor-1alpha. Stem Cells 27: 961-970, 2009. 